\title{
Smart Hybrid Nanomaterials Based on Silica- and Metal Ions/Nanoparticles Conjugates For Biosensing
}

\author{
Ignác Capek* \\ Polymer Institute, Institute of Measurement Science, Slovakia
}

*Corresponding author: Ignác Capek, Slovak Academy of Sciences, Polymer Institute, Institute of Measurement Science, Bratislava, Slovakiat

Submission: February 14, 2019; Published: February 28, 2019

\begin{abstract}
The preparation and decoration of silica-based nanomaterials have been reported. The silica-based nanomataerials and their analoques possess physical and chemical properties that strongly deviated from those typical for bulk materials. The structure of present nanostructures consists of a core and a silica shell or the silica core is coated with metal or organic shell. The shell acts as an external envelop which enhances the colloidal stability of dispersion which protects the core of the nanocrystal from photo and thermal-initiated degradation. The preparation of these hybrid nanoparticles with fluorescence (FL) properties are described. Various silica based nanomaterials have shown unique optical properties due to their large surface to volume ratio and unique electronic structure that has aided in the FL detection of various analytes. These fluorescent nanoparticles are often stable and resistant to photobleaching and have higher luminescent intensity and longer lifetime. Biodecorated hybrid nanostructures with different functional ligands used for various bioanalytical applications using FL, FRET, SPR, LSPR and SERS have been reported. The composite nanoparticles coated by organic shells with functional groups were considered to govern the covalent immobilization of biomolecules. The hybrid silica nano(bio)materials have been recently explored and are used for imaging and therapeutic applications. The nanoparticles with unique optical properties may be useful as biosensors in living whole cells. The enhanced cellular drug delivery to cancer cell lines via nanoconjugates revealed that smart nanoparticles are an effective tool for transporting and delivering drugs. Biodecorated nanoparticles provide opportunities for designing various types of therapeutics with novel properties that are not possible to obtain with traditional therapeutics.
\end{abstract}

Keywords: Composite silica-based nanoparticles; Metal nanoparticles; Biodecorated metal- and silica-based nanoparticles; Biomolecules; Application; Brug delivery and cancer therapeutics

Abbreviations: ATP: Adenosine Triphosphate; AuNPs: Gold Nanoparticle; Con A: Concanavalin A; DDSiNPs: Dye-Doped Silica Nanoparticles; EGFR: Epidermal Growth Factor Receptor; EGDN: Ethylene Glycol Dinitrate; EM: Electromagnetic; FDSiNPs: Fluorescein (Fitc)-Doped Silica Nanoparticles; FITC: Fluorescein Isothiocyanate; FL: Fluorescence; LSPR: Localized Surface Plasmon Resonance; MB: Methylene Blue; MR: Magnetic Resonance; MsiNPs: Mesoporous Silica Nanoparticles; NG: Nitroglycerin; NIR: Near Infrared; NMOFs: Nanoscale Metal-Organic Frameworks; PDMS Poly(Dimethyl-Siloxane); PDT: Photodynamic Therapy; PEI: Polyethyleneimine; PETN: Pentaerythritol Tetranitrate; PFPA: Perfluorophenyl Azide; PTT: Photothermal Therapy; RBITC: Rhodamine B Isothiocyanate; ROS: Producing Reactive Oxygen Species; SAMs: Self-Assembled Monolayers; SERS: Surface-Enhanced Raman Spectroscopy; SiHNP: Silica Hybrid Nanoparticle; SiNPs: Silica Nanoparticles; SPIO: Superparamagnetic Iron Oxide; SPR: Surface Plasmon Resonance; ssDNA: Single-Stranded DNA; TEOS: Tetraethyl Orthosilicate, tetraethoxysilane; TPA: Two-Photon Absorbing; UCNPs: Upconverting NPs

\section{Silica-Based Nanomaterials}

The types of nanomaterials include quantum dots (QD), (noble) metal nanoparticles ((N)MNPs), rare earth doped NPs (up-converting NPs), dye-doped polymer, silica and metal NPs, carbon NPs, polymeric NPs, etc. Silica-based nanomaterials, for example, such as solid silica particles and their analoques are easily prepared and used for imaging and therapeutic applications. These nanostructures can be synthesized by several methods such as oil-inwater microemulsion, surfactant-mediated hydrothermal synthesis, hydrothermal synthesis, and nanoprecipitation. Composite or hybrid nanoparticles can also be prepared by both "top down" and "bot tom up" approaches. For "top down" procedures, a bulk state silica is systematically broken down to generate nanoparticles of desired dimensions. However, the "top down "method is limited concerning the control of the size and shape of particles as well as further functionalization [1]. In contrast, in the "bottom up" strategy, the formation of NPs originates from individual molecules (atoms), because it involves a chemical or biological reduction [2]. An anisotropic snowflake-like structural assembly of polymercapped gold nanoparticles. Silica-based nanomaterials show unique properties such as high surface areas, tunable pore sizes, 
amorphous and crystalline domains, and large pore volumes. Various ligands (cargoes) can be either directly incorporated in the mesoporous silica matrix or grafted to the outer surface of the solid silica nanoparticles (SiNPs) via their functional groups. The functionalized SiNPs can be decorated with some agents in several ways, including loading of cargo into the pores, covalent grafting, and co-condensation of siloxy-derived cargoes.

Two major strategies are used to synthesize silica nanoparticles: the sol-gel synthesis and reverse microemulsion synthesis (Figure $1 \& 2$ ). The sol-gel synthesis of monodisperse solid silica particles ranging in size from $50 \mathrm{~nm}$ to $2 \mu \mathrm{m}$ was reported by Stöber and coworkers [3]. This method involves the controlled hydrolysis and condensation of a silica precursor, such as tetraethyl orthosilicate (TEOS), in ethanol using ammonia as a catalyst (Figure 2, top). The size of the particles can be tuned by adjusting the reaction conditions. For example, adjusting the TEOS concentration from
$0.05 \mathrm{M}$ to $0.67 \mathrm{M}$ while keeping the other reactant conditions constant affords silica particles from 20 to $880 \mathrm{~nm}$ in size [4]. These particles remain stable in solution due to electrostatic repulsion from the negatively charged silica particles. Another common method for the synthesis of monodisperse silica nanoparticles uses reverse phase, or water-in-oil, microemulsions (Figure $1 \& 2$, bottom) [5,6]. Reverse microemulsions consist of nanometer-sized water droplets stabilized by a surfactant in a predominantly organic phase. The aqueous droplets in the microemulsion essentially act as "nanoreactors" that assist in controlling the kinetics of particle nucleation and growth. The precursor TEOS is hydrolyzed at the micellar interface and enters the aqueous droplet to form a silica nanoparticle within the microdroplet. The size and number of emulsified droplets (suppramicelles) within the microemulsion can be tuned by varying the water to surfactant ratio $(\omega)$. This method allows for careful control of particle size (much below 100nm) and polydispersity.

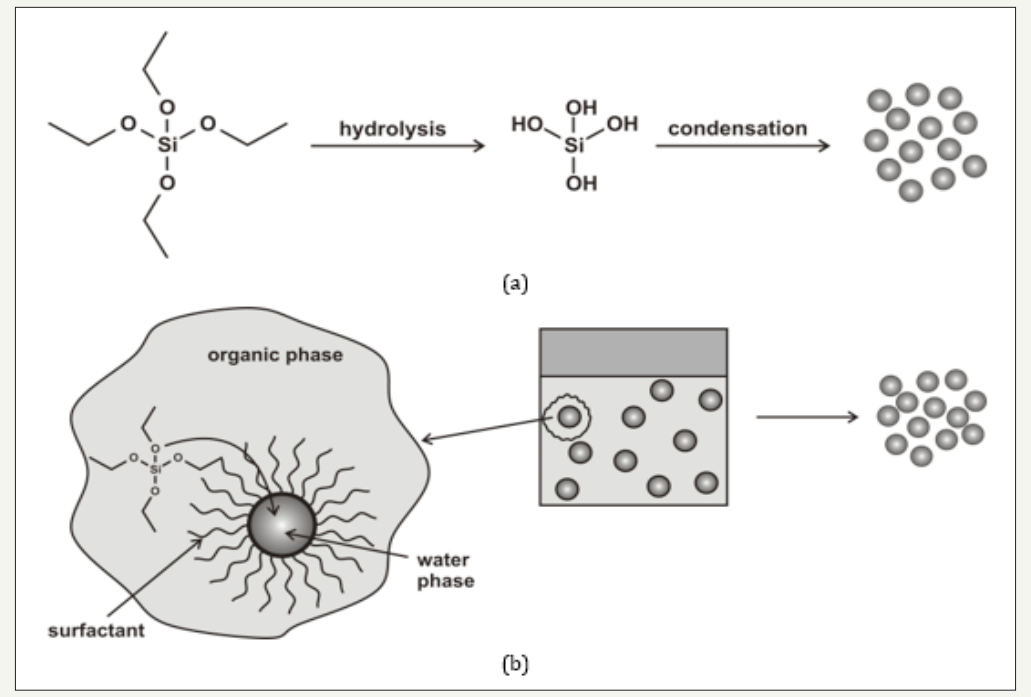

Figure 1: Methods for synthesizing solid silica nanoparticles. (a) The Stöber method, (b) The reverse phase microemulsion

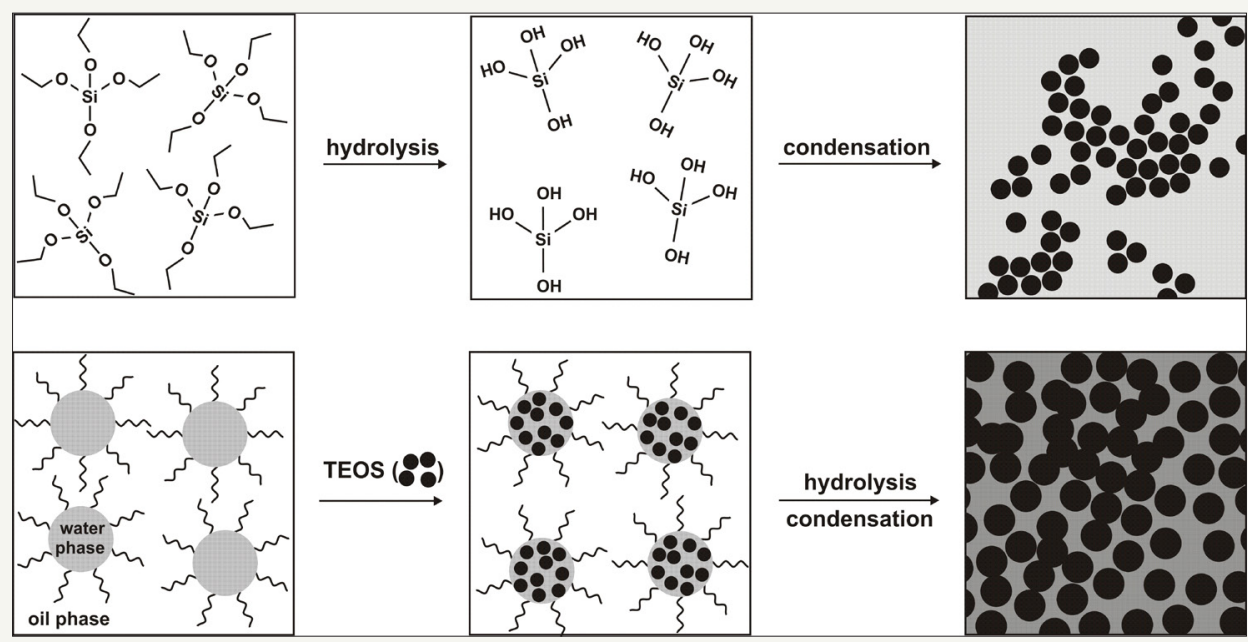

Figure 2: Methods for synthesizing solid silica nanoparticles. (Top) The Stöber method, (bottom) The reverse phase microemulsion [6].c 
The previous synthetic ways can be used to prepare the silicabased hybrid nanomaterials: solid silica particles and mesoporous silica nanoparticles (MSiNPs). Unlike solid silica particles, MSiNPs exhibit many unique properties such as high surface areas, tunable pore sizes, and large pore volumes. The imaging or therapeutic cargoes can be either directly incorporated in the silica matrix or grafted to the outer surface of the solid silica particles. MSiNPs can be functionalized with imaging or therapeutic agents in several ways, including loading of cargo into the pores, covalent grafting, and co-condensation of siloxy-derived cargoes.

Grafting to the outer surface of the silica nanoparticles (SiNPs) via their functional groups can be prepared interesting dyedoped silica nanoparticles (DDSiNPs). DDSiNPs consist of silica matrix NPs doped with a large number of dye molecules inside [7]. Compared to QDs, silica nanoparticles are not toxic, the silica shell can prevent the dyes from photobleaching, they are highly hydrophilic, and they are photophysically inert since they are not involved in the absorbance and emission processes [8]. The silica nanoparticles are reported to be mostly prepared by the Stöbermethod, using monomeric tetraethoxysilane (TEOS) as a precursor to the silica and organic agents which are covalently linked to the highly porous silica matrix [9]. The inverse microemulsion method offers relatively easier control over the size of the silica NPs [10]

The optical properties of the hybrid nanoparticles (e.g., dye-, silver-, gold-, etc. doped silica nanoparticles) are controlled by the amount and properties of the (molecular) agents inside and outside. Shi and coworkers described $\mathrm{pH}$ nanosensing with dye-doped silica NPs (DDSiNPs) that were synthesized by a reverse microemulsion method and doped with a pH sensitive squaraine dye [10]. The authors modified the surface of the NPs with carboxyl groups to avoid aggregation of the particles and increase water solubility. They optimized the amount of dye molecules doped inside the silica particles for optimal sensitivity while also decreasing selfquenching. The intensity of the emission spectra increased linearly with increasing $\mathrm{pH}$. Many other ions, including alkali, alkaline earth, and transitional metal ions do not significantly interfere with the performance of this $\mathrm{pH}$ sensor.

Since hydrophilic DDSiNPs exhibit excellent biocompatibility, they have accordingly found use in biological sensing and imaging. Wang et al. [11] demonstrated the labeling of glycan by DDSiNPs. The authors report that dye-doped fluorescent silica nanoparticles (DDFSiNPs) are highly efficient labels for glycans. Mono- and oligo-saccharides were further conjugated to DDFSiNPs using a general photocoupling chemistry. DDFSiNP-labeled glycans were applied to image and detect bacteria, and to study carbohydratelectin interactions on a lectin microarray. Thus, they also reported using DDFSiNP labeled glycan to image and detect bacteria and to study glycan-lectin interactions on a lectin microarray Fluorescein (FITC)-doped silica nanoparticles (FDSiNPs) $\sim 100 \mathrm{~nm}$ in diameter were synthesized by a modified Stöber-method and functionalized with perfluorophenyl azide (PFPA) silane because PFPA can be photochemically or thermally activated to singlet perfluorophenylnitrene. PFPA-FDSiNP was labeled with different carbohydrates by incubating in microvials with UV irradiation.
The resulting solution became highly fluorescent. They tested the activity of the FDSiNP-carbohydrates (FSNP-mannose and FDSiNPgalactose) by treating them with Concanavalin A (Con A), which specifically binds with mannose. This results in the agglomeration of the nanoparticles and a corresponding decrease in fluorescence (FL) intensity of the NPs. Since Con A does not bind with galactose, the emission spectra did not change. The authors applied these FDSiNPs to the biological imaging and detection of E coli. They treated the FDSiNP-mannose with two bacteria strain; one had a mannose specific binding domain while the other did not. The first sample showed a strong FL intensity while the second did not exhibit FL. They also studied the carbohydrate-lectin interaction on a lectin microarray, using this glycan label. The regions with Con A showed much higher FL intensity than those treated with a nonmannose binding lectin. This glycan labeling method is simple and effective, and the labeled glycan maintains its specificity and reactivity.

Zhang and co-workers described FRET-based ratiometric $\mathrm{pH}$ sensing using dye pair doped mesoporous silica nanoparticles [12]. Incorporation of a dual-FRET dye pair into mesoporous silica nanoparticles yields sensitive and sensing-range tunable nanosensors with good reversibility that can be used for ratiometric $\mathrm{pH}$ measurements under a single-wavelength excitation. Fluorescein isothiocyanate (FITC) and rhodamine B isothiocyanate (RBITC) were codoped into the NP, which served as the FRET donor and acceptor, respectively. RBITC quenched the emission of the FITC (at $521 \mathrm{~nm}$ ) and emitted a FRET signal at $575 \mathrm{~nm}$. The emission signal intensity of FITC increased with increasing $\mathrm{pH}$ value while the intensity of the FRET signal remained the same. By monitoring the intensity ratio of IFITC/RBITC as a function of $\mathrm{pH}$ with different ratios of the two dyes doped in the NPs, the authors discovered that the $\mathrm{pH}$ sensing range varied with different doping ratios and that the largest range from $\mathrm{pH}$ 4-9 occurred with a FITC-RBITC of 1:2. The detection sensitivity also varied with different doping ratios, where a FITC-RBITC of 4:1 was most sensitive. The authors attribute this to the increased interaction of $\mathrm{H}^{+}$with FITC at high local concentrations.

Lu [13] covalently linked RBITC on the surface of silica NPs with FITC-doped in the interior. The FITC served as a reference dye and RBITC as a response dye. These NPs were also functionalized with polyethyleneimine (PEI) as a stabilizer and chelating agent for the detection of $\mathrm{Cu}^{2+}$ in water samples and cells. The NPs emitted at 518 and 580nm for FITC and RBITC, respectively. The RBITC emission band decreased through quenching in the presence of $\mathrm{Cu}^{2+}$ due to its binding to the surface while the reference FITC was unaffected. $\mathrm{Ag}^{+}$and $\mathrm{Hg}^{2+}$ affected the FL intensity of the NPs. The addition of $\mathrm{NaCl}$ recovered the $\mathrm{FL}$ signal because $\mathrm{Cl}^{-}$precipates with $\mathrm{Ag}^{+}$and forms complexes with $\mathrm{Hg}^{2+}$. $\mathrm{Co}^{2+}$ and $\mathrm{Ni}^{2+}$ can form complexes with PEI at high concentrations, which can be overcome by the addition of $\mathrm{HNO}_{3}$. Acidic conditions do not affect the response to $\mathrm{Cu}^{2+}$ significantly due to the high affinity of PEI to $\mathrm{Cu}^{2+}$. The authors imaged intracellular $\mathrm{Cu}^{2+}$ with confocal laser scanning microscopy by incubating HeLa cells in the nanoprobe solution and exposing to $\mathrm{Cu}^{2+}$. 
Kim and coworkers have reported the preparation of novel silica-based hybrid nanocomposite. They used several polymers to decorate silica nanoparticles for photodynamic (PDT) therapy [14]. PDT is a light-activated treatment for cancer and other diseases. This approach is based on utilizing light sensitive agents located at or within the tumor tissues. Therapeutic effect of light sensitive drugs is based on their photoexcitation and formation of radical fragments. In some cases photosensitizer is used to increase the quatum yield of radical formation. The transfer of the energy from the photosensitizer to some species can generate cytotoxic species such as singlet oxygen $\left({ }^{1} \mathrm{O}_{2}\right)$. The generated radicals interact with the unsaturated bonds of malignant cells (tissues) and destruct them. The generated radicals (cytotoxic species) initiate the selective destruction of diseased cells, without destruction of adjacent healthy ones. However, traditional PDT photosensitizers absorb in the visible spectral region below $700 \mathrm{~nm}$, where light penetration into the skin is only a few millimeters. Under such conditions the generation of cytotoxic (excited states) species is low or negligible. This drawback can be overcome by using mixed photo-senstitive systems, for example, combining a two-photon absorbing (TPA) dye with the photosensitizer [6]. Herein, the photosensitizer is indirectly excited through fluorescence resonance energy transfer (FRET), that is, the donor absorbs light and then the transfer of energy to acceptor leads to the exciation of PDT photosensitizers. A photosensitizer and a two-photon energy donor can be together co-encapsulated in a silica- based nanocomposite (Eqs. 1 and 2). Upon two-photon irradiation, the two-ways transfer of energy proceeds due to which the photosensitizer is excited as a result of intraparticle FRET from the two-photon absorbing dye. The final result of this transfer energy in the generation of excited species especially singlet oxygen. The uptake of hybrid silica nanoparticles was followed via fluorescence history imaging of malignant HeLa cells. The malignant cells via two-photon irradiation of the nanoparticle conjugates is accompanied by drastic morphology changes. The cell necrosis, apparently is induced by the reactive oxygen species $\left({ }^{1} \mathrm{O}_{2}\right)$ generated by the photosensitizer:

${ }^{3} \mathrm{O}_{2}$-..- photosensitizer with the light of $\lambda<700 \mathrm{~nm} \rightarrow{ }^{1} \mathrm{O}_{2}$ (slight cytotoxicity), the light penetrates into the skin only a few millimeters

${ }^{3} \mathrm{O}_{2}$ _._- photosensitizer/ FRET with TPA dye $\rightarrow{ }^{1} \mathrm{O}_{2}$ (strong cytotoxicity), (2)

the light penetrates into the skin deeply and therefore the drastic morphology changes associated with cell necrosis were observed

This strategy was used by Hai and coworkers who have prepared silica nanoparticles modified with methylene blue (MB) dye ligands [15]. This silica-based nanoconjugate was used for near-IR (NIR) imaging and PDT. The optical properties of the excited MB molecules and their photoinduced transient species and fragments were studied by time-resolved near infrared (NIR) emission (efficiency of singlet oxygen generation), by laser flash photolysis (transient spectra, yield and decay rate of triplets) and by thermal lensing (amount of heat deposited in the medium) to get the whole history of present photolysis including the electron transfer and energy transfer processes and their competition. Among effective photosensitizers with their high quantum yield of ${ }^{1} \mathrm{O}_{2}$ generation belongs methylene blue. $\mathrm{MB}$ is also characterized with low dark toxicity, and NIR therapeutic window. PDT with PD as photosensitizer was applied in vitro against HeLa cells. Significant cell death was only observed in cells treated with the MB hybrid nanoparticles after laser irradiation. The negligible toxicity against malignant cells was observed with either the nanoparticles or the laser alone. This can be discussed in terms of excitation and electron transfer reactions of $\mathrm{MB}^{*}$ with tumor tissue matrix. After intratumoral treatment with excited injection $\mathrm{MB}^{*}$-nanoconjugate the tumor became necrotic.

Lin et al. [16] describe the synthesis of a nano-porous multilayered film consisting of $\mathrm{Au} @ \mathrm{SiO}_{2}$ nanoparticles [16]. This film was used to miniaturize the size of a localized surface plasmon resonance (LSPR)-based capillary gas chromatograph (GC) detector. A layer-by-layer (LbL) approach with proper surface reaction sequences was used to create a multilayer structure that consisted of as many as five layers of $\mathrm{Au} @ \mathrm{SiO}_{2}$ nanoparticles. The center wavelength of LSPR was shifted from 520 to $634 \mathrm{~nm}$ due to the approximation of additional layers of nanoparticles. The vapor response time for this $\mathrm{Au} @ \mathrm{SiO}_{2}$ multilayer LSPR sensor was identical to that of an Au nanoparticle monolayer, which confirmed that this multilayer structure has a high level of gas permeability. The multilayer was synthesized inside a glass capillary for use as a GC detector. Due to the enhancement of absorbance, the gas chromatographic signal was obtained via a single spotlight that penetrated one side of the glass capillary and was then reflected by a silver mirror coated on the opposite side. The detection limits were $\leq 20 \mathrm{ng}$ for cyclohexanone and m-xylene.

Silica hybrid nanoparticles (SiHNPs) - DNA bioconjugates can be used as a template for DNA carriers for various therapy treatments. Fluorescently labeled SiHNPs stabilized or covered with cationic suractants or cationic surface coatings are able to chemisorb oligo DNAs [17]. The DNA-based bioconjugates are formed by the electrostatic interaction between negatively charged DNA and positively charge particle surface coating. Furthermore, the conjugate structure prevents enzymatic degradation of the DNA ligand (supported by the gel electrophoresis data). The biocompatibility of SiHNP-DNAs nanoconjugates led to the high entry rate into the cells in vitro accompanied with the released DNA to the nucleus (supported by confocal microscopy data). Similarly other DNA-based decorated silica nanoparticle structures were able to tune activities of neural cells [18].

Yan et al. [19] have decorated the silica nanoparticles with the chitosan [19]. The SiNP-chitosan conjugate uptakes the anticancer drug doxorubicin (Dox). Fabricated SiNP-Dox nanoconjugates were found to be $\mathrm{pH}$ sensitive with the minimal drug release at $\mathrm{pH}=$ 7.4. They exhibit desired rapid release of the drug at lower $\mathrm{pH}$ at ca. 4. Cytotoxicity assay studies demonstrated that the bound Dox within the nanoconjugate was much more effective than free Dox, presumably by means of more efficient drug uptake and its safe 
transfer. Corma et al. [20] have synthetisized similar nanoconjugate consisting of soft liposomal nanoparticle template saturated with doxorubicin and coated by silica domains [20]. These drag saturated nanoconjugates exhibit desired interaction with human glioma cells in vitro assays with increased cell death. Fluorescent imaging of the cells revealed that the interaction of nanoconjugates with the cell surface (receptors) favours first the releasing of drug and second its entry into the cells.

Mesoporous silica nanoparticles belong among functional nanostructures with a high surface area and tunable pore structures exhibiting high delivery activities for various therapeutics. The MCM-41 type of MSiNPs possess tunable pore diameters from 2-10nm convenient for the drug uptake [21]. Lin et al. [16] have a used the MCM-41-type nanoparticles the delivery of various bioagents such as a membrane impermeable protein, cytochrome C, etc [22]. After reorganization of surfactant molecules on the particle surface and within the pores, the physi- and chemisorbed proteins and cytochrome $\mathrm{C}$ could be efficiently uptaken by the particle surface pores. The $\mathrm{pH}$ sensitivity was applied to regulate the release of the additive at a given site as shown: 45 and 55\% protein was released at $\mathrm{pH}$ values of 7.4 and 5.2, respectively. This behavior was discussed in terms of variation of the negative charge on silica nanoparticles with the $\mathrm{pH}$ values due to which varies the interaction of MSNPs with the positively charged protein. The bioconjugate MCM-41 was efficietly uptaken with HeLa cells. The absortion (uptake)/release was revealed for the proteins from endosomal entrapment proved by confocal microscopy. Similarly, the MSiNP - cystein conjugate was used to deliver cysteine to various cells [23]. The chemisorbed cysteine to the MSiNP can be only released in the presence of reducing agent. Thus, the cysteins bound on or within MSiNPs were 440 times more effective at inhibiting cell growth (the nonconventional therapy) than free $\mathrm{N}$-acetylcysteine (the standard cysteine therapy).

Lai and coworkers have synthesized several MSiNPs-based nanoconjugates containing stimuli-responsive pore agents (such as $\mathrm{CdS}, \mathrm{Au}$ and $\mathrm{Ag}$ ions and nanoparticles, superparamagnetic iron oxide (SPIO) nanoparticles, etc.) for controlling the delivery and release of entrapped cargoes [24]. The AuNP-MSiNP-paclitaxel nanoconjugate, for example, with a photolabile linker which is responsible for the release of the drug when exposed to UV light, was used for the drug delivery. This approach was used for the deliver of other anticancer drugs such as paclitaxel-based derivatives (human liver and fibroblast cells), doxorubicin, vancomycin, adenosine triphosphate (ATP) and so on. After UV irradiation, a photolabile linker was broken and free drugs decreased the cell viability. The selection of UV irradiation conditions anabled transfer and release of discussed drugs and neurotransmitters to the specific sites and tissues [24].

Hyeon and coworkers synthesized composite nanoparticles with a iron oxide nanoparticle core and a mesoporous silica shell, that is, the core@shell structure [25]. SPIO nanoparticles provide $\mathrm{T}_{2}$-weighed magnetic resonance (MR) contrast enhancement, while the silica shell serves as a matrix for functionalization. The nanoconjugate can carry on the surface chemisorbed fluorescent dye molecules and the anticancer drug doxorubicin is exfoliated within the pores. In vitro assay experiment again confirmed that the bare nanoparticles possessed minimal cytotoxicity to cancer cells, while the complexed doxorubicin within the particle nanoconjugate possessed a dose dependent cytotoxicity profile. In vivo assays the $\mathrm{T}_{2}$ signal trongly decreased at the tumor site after injection of drug containing nanoconjugates, which results from the ex vivo organ analysis by fluorescence microscopy.

Zink et al. have described the preparation magnetic nanoconjugate consisting of SPIO nanocrystal cores and silica shell modified with hydrophilic ligands to prevent aggregation. As in the previous study the conjugate was also labeled with fluorescent dye molecules for fluorescent imaging of the cells [26]. Herein the water-insoluble anticancer drugs (camptothecin and paclitaxel) was delivered via nanoparticle conjugate into human cancer cells. Consequenly, the nanoconjugates increased the uptake of drug entities into cancer cells.

Lai et al. [27] have constructed magnetic conjugates on the SPIOand silica-based nanomaterials coated SPIO for both a multimodal system for MR imaging and photodynamic therapy [27]. In order to increase their photodynamic activities the nanoconjugates were inreached with an iridium photosensitizer. HeLa cells were selected for further studies using confocal microscopy while they efficiently uptake magnetic nanoconjugates. The nanoconjugates also exhibited desired $\mathrm{T}_{2}$-weighted contrast and signal reduction. The cytotoxicity assay synergism appeared only with the UV light irradiation, that is, the cell death rate strongly increased with irradiation.

Je and co-workers fabricated rhodamine B coated AuNP arrays on an elastomeric substrate of poly(dimethyl-siloxane) (PDMS) and monitored the FL intensity change as a function of pressure [28]. With an increase in pressure, the elastic substrate became compressed and the distance between the AuNPs decreased, resulting in an increase in the FL intensity. The FL intensity decreased after relief of the pressure.

\section{Metal-Based Nanomaterials}

Composite nanomaterials based on (noble) metal nanoparticles are fascinating materials with great nanotechnological potential due to their unique and strongly sizedependent electronic, optical, physical, and chemical properties [29]. Additionally, the surfaces of nanoparticles can be easily functionalized with various organic and biomolecular ligands, among which the molecules with a sulfur and silica headgroup are attracting considerable interest [30]. The strong affinity of sulfur to gold and silver has been exploited to form molecular contacts, to link other species to the gold surface, or to form well-ordered self-assembled monolayers (SAMs) [31] for applications like surface patterning [32]. Additionally, nanoparticles can be fashioned with a wide range of metal and semiconductor core materials that impart useful properties such as fluorescence and magnetic behavior [33]. Nanoparticles belong to a multidisciplinary field, which covers a vast and diverse array 
of devices derived from engineering, biology, physics and chemistry. These devices include nanovectors for the targeted delivery of anticancer drugs and imaging contrast agents. Nanowires and nanocantilever arrays are among the leading approaches under development for the early detection of precancerous and malignant lesions from biological fluids. These and other nanodevices can provide essential breakthroughs in the fight against cancer. The interaction of light with noble metal nanoparticles results in collective oscillations of the free electrons in the metal known as localized surface plasmons (SPR and LSPR). On resonance, a metallic nanoparticle interacts strongly with incident light, possessing an extinction cross section nominally five times its physical cross section.

We highlight advantages and disadvantages of functionalized nanoparticles through various detection modes, including colorimetry, fluorescence, electrochemistry, SPR, and mass spectrometry for the detection of small molecules, oligonucleotides and proteins. The functionalized nanoparticles are selective and sensitive for the analytes, showing their great potential in biosensing. Furthermore, this contribution shortly reviews recent progress in the area of DNA- metal nanoparticles based artificial nanostructures. The preparation, collective properties, and applications of various DNA-based nanostructures are also important.

It was reported that Apt-AuNPs have been used in SERS for thrombin detection. Raman scattering cross-section of a molecule in SERS spectroscopy based on molecules residing at or near the surface of certain nanostructured metals can be increased by factors up to $10^{14}-10^{15}$, that is comparable to fluorescence [34]. This great enhancement is presumably from the large electromagnetic (EM) field produced by hot spots, which reside in the nanoscale junctions or interstices in metal nanostructures such as dimers or aggregates. Moreover, SERS has the inherent advantages over fluorescence that narrower bandwidth and provide richer spectral information.

Addition of the complementary fluorescein-labeled oligonucleotide in the presence of the silver nanoparticles resulted in a 12 -fold increase in the fluorescence intensity. The enhanced steady state fluorescence was attributed to the shortening in the lifetime of the dye as it entered into proximity of the AgNPs. Thus, hybridization could be followed by the analysis of the decay kinetics of the emission of the dye. These results suggest that silver particles could be used as a versatile label for the amplified detection of DNA hybridization. Similarly, silver nanoparticle-enhanced fluorescence was applied in the immunoassay of insulin in serum [35].

The metal nanoparticles functionalized with various groups represent smart and promising candidates in the drug delivery applications due to their unique dimensions, tunable functionalities on the surface and controlled drug release [36]. Another essential aspect while working with AuNPs and/or AgNPs in bio-applications is safety and biocompatibility. Biologically synthesized and functionalized, AuNPs provide many desirable attributes for use as carriers in drug delivery systems as the functionalized AuNP core is essentially inert and nontoxic [37]. These functionalized nanodelivery particles can be used directly as promising agents in the detection of cancer cells. The use of synthesized bio-functional noble metal nanoparticles as an anti-cancer drug demonstrates the anti-cancer effect of these functionalized AuNPs. It gives a strong speculation, for the influence of free electrons generated by the surface of the functionalized AuNP has a lethal effect on the electronegative surface membrane of the cancer cells.

Nanoshells can be composed of various materials. The core surrounded by a thin gold shell will absorb energy (heat up) when exposed to the appropriate wavelength of light. The near-infrared characteristics were chosen because absorption by tissues is minimal and penetration of the light is optimal at this wavelength. The nanoshells can accumulate in its "leaky" and will allow nanosized particles to penetrate into the tumor while normal tissue or organs are not affected. The tumors are then illuminated with a near-infrared diode laser to heat the tumor and cause cellular destruction. By this protocol all the tumors were ablated, and the mice remained tumor-free for many months. Although not used in these studies, the efficacy of the procedure could be improved by attachment of tumor-homing or -targeting molecules to the nanoshells for increasing concentration at the site of heating. In appropriate settings, thermal ablation methods could be used to replace surgical resection of tumors, and targeted therapies and immunotherapy as a substitute for toxic. This approach has been recently developed and successfully used for ablation therapy in vitro and in vivo. It is becoming increasingly clear that a bonetwo punch is needed to eradicate tumors, and the nanomedicinal methods being developed have a good chance of achieving this goal with much less damage to normal tissue than existing therapeutic protocols [38].

Noble metal-based structures have been of great interest in biomedical applications. Among these metals, gold-based systems provide a unique combination of properties. Gold is resistant to oxidation under physiological or ambient conditions, which permits unrestricted interaction of gold with the biological environment and also provides flexible ways of modifying the Au surface with various bioactive molecules under a variety of chemical conditions. Moreover, gold surfaces and Au-based nanoscale structures exhibit unique optical properties, particularly surface plasmon resonance in the visible range of the electromagnetic spectrum; the optical properties are also dependent on the particle shape and size, which provide a way to tune optical information (e.g., color) based on particle dimensional parameters. In addition, light absorbed by the $\mathrm{Au}$ plasmonic process is converted to heat, which can be exploited in developing various photothermal therapy (PTT) strategies. Thus, photothermal ablation therapy is based upon application of gold "nanoheaters" able to absorb laser light near their plasmon resonance band, with subsequent heat generation. Although such direct PTT application has been demonstrated mostly in the context of thermal ablation of tumors, one can envision the design of vascular drug-loaded multicomponent nanoconstructs 
where one of the components is photoresponsive gold, such that upon inducing photothermal response, the Au components help in thermal destabilization of the nanoconstruct and release of the encapsulant. It is to be noted that photothermal or laser-based ablation phenomena may actually damage the vascular endothelium and lead to secondary thrombotic and inflammatory events. The general scheme of "classical" PDT includes three principal components: photosensitizers (PSs) or natural dye precursors (such as $\delta$-aminolevulinic acid), light and oxygen. The absorption of light energy by the PS is followed by energy dissipation from the excited state via two major pathways: 1) the emission of fluorescence suitable for cancer detection, and 2) energy transfer to molecular triplet oxygen, producing reactive oxygen species (ROS) able to photooxidize bioorganic molecules and terminate cancer cells. Nearly $100 \%$ absorbed light is converted to heat via the nonradiative properties. AuNPs or silica doped AuNPs structures are very photostable and biocompatible. These features make them a new generation photothermal contrast agents for PTT, in which photon energy is converted to heat sufficient to induce cellular damage via thermal effects such as hyperthermia, coagulation and evaporation. In addition, approaches utilizing other photonic hybrid materials based on $\mathrm{Au}, \mathrm{Ag}, \mathrm{Si}$, etc. were reported to be useful for photothermal ablation therapy of brain cancer cells. In spite of the diversity of chemical composition, structure and mechanism of action, all PS approaches share one common requirement-they must be inducible in a spectral window near the optimal biological optical area. Thus PTT is an experimental use of electromagnetic radiation which has been used to treat various medical conditions, including cancer. Cancer patients without any other alternative for treatment, now have a choice, to treat their cancer using nanophotothermolysis. Unique properties such as absorption and scattering of electromagnetic radiation have been used in photothermal therapy. PTT using spherical gold-based nanostructures can be achieved with pulsed or cw visible lasers due to the SPR absorption in the visible region and thus such treatment is suitable for shallow cancer (e.g. skin cancer) [39,40].

Noble metal, especially gold $(\mathrm{Au})$ and silver $(\mathrm{Ag})$ nanoparticles exhibit unique and tunable optical properties on account of their surface plasmon resonance (SPR) [41]. Herein, we discuss the SPRenhanced optical properties of noble metal nanoparticles, with an emphasis on the recent advances in the utility of these plasmonic properties in molecular-specific imaging and sensing, photodiagnostics, and selective photothermal therapy. The strongly enhanced SPR scattering from Au nanoparticles makes them useful as bright optical tags for molecular-specific biological imaging and detection using simple dark-field optical microscopy. On the other hand, the SPR absorption of the nanoparticles has allowed their use in the selective laser photothermal therapy of cancer. We also mention the sensitivity of the nanoparticle SPR frequency to the local medium dielectric constant, which has been successfully exploited for the optical sensing of chemical and biological analytes. Plasmon coupling between metal nanoparticle pairs is also mentioned, which forms the basis for nanoparticle assembly-based biodiagnostics and the plasmon ruler for dynamic measurement of nanoscale distances in biological systems.

Nanostructures attract much interest because of their unique properties, including large optical field enhancements resulting in the strong scattering and absorption of light [42]. The enhancement in the optical and photothermal properties of noble metal nanoparticles arises from resonant oscillation of their free electrons in the presence of light, also known as localized surface plasmon resonance (LSPR). The plasmon resonance can either radiate light (Mie scattering), a process that finds great utility in optical and imaging fields, or be rapidly converted to heat (absorption); the latter mechanism of dissipation has opened up applications in several new areas.The ability to integrate metal nanoparticles into biological systems has had greatest impact in biology and biomedicine. In this account, we discuss the plasmonic properties of gold and silver nanostructures and present examples of how they are being utilized for biodiagnostics, biophysical studies, and medical therapy. For instance, taking advantage of the strong LSPR scattering of gold nanoparticles conjugated with specific targeting molecules allows the molecule-specific imaging and diagnosis of diseases such as cancer. We emphasize in particular how the unique tunability of the plasmon resonance properties of metal nanoparticles through variation of their size, shape, composition, and medium allows chemists to design nanostructures geared for specific bio-applications. There are some interesting nanostructure geometries, including nanorods, nanoshells, and nanoparticle pairs, that exhibit dramatically enhanced and tunable plasmon resonances, making them highly suitable for bio-applications. Tuning the nanostructure shape (e.g., nanoprisms, nanorods, or nanoshells) is another means of enhancing the sensitivity of the LSPR to the nanoparticle environment and, thereby, designing effective biosensing agents. Metal nanoparticle pairs or assemblies display distance-dependent plasmon resonances as a result of field coupling. A universal scaling model, relating the plasmon resonance frequency to the interparticle distance in terms of the particle size, becomes potentially useful for measuring nanoscale distances (and their changes) in biological systems. The strong plasmon absorption and photothermal conversion of gold nanoparticles has been exploited in cancer therapy through the selective localized photothermal heating of cancer cells. For nanorods or nanoshells, the LSPR can be tuned to the near-infrared region, making it possible to perform in vivo imaging and therapy.The examples of the applications of nanostructures based on noble metals provided herein can be readily generalized to other areas of biology and medicine because plasmonic nanomaterials exhibit great range, versatility, and systematic tunability of their optical attributes.

Due to strong electric fields at the surface, the absorption and scattering of electromagnetic radiation by noble metal nanoparticles are strongly enhanced [43]. These unique properties provide the potential of designing novel optically active reagents for simultaneous molecular imaging and photothermal cancer therapy. It is desirable to use agents that are active in the near-infrared (NIR) region of the radiation spectrum to minimize the light extinction 
by intrinsic chromophores in native tissue. Gold nanorods with suitable aspect ratios (length divided by width) can absorb and scatter strongly in the NIR region (650-900nm). Herein, we provide an in vitro demonstration of gold nanorods as novel contrast agents for both molecular imaging and photothermal cancer therapy. Nanorods are synthesized and conjugated to anti-epidermal growth factor receptor (anti-EGFR) monoclonal antibodies and incubated in cell cultures with a nonmalignant epithelial cell line (HaCat) and two malignant oral epithelial cell lines (HOC 313 clone 8 and HSC 3). The anti-EGFR antibody-conjugated nanorods bind specifically to the surface of the malignant-type cells with a much higher affinity due to the overexpressed EGFR on the cytoplasmic membrane of the malignant cells. As a result of the strongly scattered red light from gold nanorods in dark field, observed using a laboratory microscope, the malignant cells are clearly visualized and diagnosed from the nonmalignant cells. It is found that, after exposure to continuous red laser at $800 \mathrm{~nm}$, malignant cells require about half the laser energy to be photothermally destroyed than the nonmalignant cells. Thus, both efficient cancer cell diagnostics and selective photothermal therapy are realized at the same time.

A novel biosensor array for DNA detection based on the coupling of silver nanoparticles is developed [44]. Two kinds of DNA-silver nanoparticle conjugates were immobilized on the aldehyde modified slides. The fluorescence intensity of Cy3 within the proximity of the conjugates was enhanced due to the coupling of the silver nanoparticles based on hybridization of target DNA and the conjugates. The fluorescence intensity increases with the concentration of target DNA, and the enhancement factor is about 2.4 at the optimized conditions. The results gave a good linear correlation between DNA concentration and fluorescence intensity at DNA concentration range from $12.8 \mathrm{pM}$ to $40 \mathrm{nM}$. The biosensors constructed on the slide as a microarray offer a unique approach for DNA detection with the advantages of high sensitivity and rapidity. The fluorescence enhancement was attributed to the coupling between the silver nanoparticles. This new strategy opens the possibility for the preparation of highly enhanced plasmonic substrate for DNA analysis.

\section{Conclusion}

Silica-based nanomaterials, for example, such as solid silica particles and their analoques are easily prepared and used for imaging and therapeutic applications. These nanostructures can be synthesized by several methods such as oil-in-water microemulsion, surfactant-mediated hydrothermal synthesis, hydrothermal synthesis, and nanoprecipitation. The functionalized SiNPs can be decorated with some agents in several ways, including loading of cargo into the pores, covalent grafting, and co-condensation of siloxy-derived cargoes. The sol-gel synthesis of monodisperse solid silica particles ranging in size from $50 \mathrm{~nm}$ to $2 \mu \mathrm{m}$ was reported. The size and number of emulsified droplets (suppramicelles) within the microemulsion can be tuned by varying the water to surfactant ratio $(\omega)$. This method allows for careful control of particle size (much below 100nm) and polydispersity. Silica particles exhibit many unique properties such as high surface areas, tunable pore sizes, and large pore volumes. The imaging or therapeutic cargoes can be either directly incorporated in the silica matrix or grafted to the outer surface of the solid silica particles. MSiNPs can be functionalized with imaging or therapeutic agents in several ways, including loading of cargo into the pores, covalent grafting, and cocondensation of siloxy-derived cargoes. Compared to QDs, silica nanoparticles are not toxic, the silica shell can prevent the dyes from photobleaching, they are highly hydrophilic, and they are photophysically inert since they are not involved in the absorbance and emission processes. It was reported that the preparation of novel silica-based hybrid nanocomposite can be applied for photodynamic (PDT) therapy. PDT is a light-activated treatment for cancer and other diseases. This approach is based on utilizinglight sensitive agents located at or within the tumor tissues. Therapeutic effect of light sensitive drugs is based on their photoexcitation and formation of radical fragments. The generated radicals interact with the unsaturated bonds of malignant cells (tissues) and destruct them. The silica-based methylene blue nanoconjugate was used for near-IR (NIR) imaging and PDT. NIR therapeutic window was applied in vitro against HeLa cells. Significant cell death was only observed in cells treated with the MB hybrid nanoparticles after laser irradiation. The SiNP-chitosan conjugate uptakes the anticancer drug doxorubicin. Fabricated SiNP-Dox nanoconjugates were found to be $\mathrm{pH}$ sensitive. Mesoporous silica nanoparticles belong among functional nanostructures with a high surface area and tunable pore structures exhibiting high delivery activities for various therapeutics. MSiNPs-based nanoconjugates containing stimuli-responsive pore agents (such as $\mathrm{CdS}, \mathrm{Au}$ and $\mathrm{Ag}$ ions and nanoparticles, superparamagnetic iron oxide (SPIO) nanoparticles, etc.) were used for controlling the delivery and release of entrapped cargoes . The AuNP-MSiNP-paclitaxel nanoconjugate, for example, with a photolabile linker which is responsible for the release of the drug when exposed to UV light, was used for the drug delivery. This approach was used for the deliver of other anticancer drugs such as paclitaxel-based derivatives (human liver and fibroblast cells), doxorubicin, vancomycin, adenosine triphosphate, etc. Gold nanorods with suitable aspect ratios (length divided by width) can absorb and scatter strongly in the NIR region (650$900 \mathrm{~nm}$ ). Herein, we provide an in vitro demonstration of gold nanorods as novel contrast agents for both molecular imaging and photothermal cancer therapy. Magnetic nanoconjugate consisting of SPIO nanocrystal cores and silica shell modified with hydrophilic ligands deliver via nanoparticle conjugatedrugs into human cancer cells. We highlight advantages and disadvantages of functionalized metal nanoparticles through various detection modes, including colorimetry, fluorescence, electrochemistry, SERS, LSPR, QDs, FRET, SERS, etc. for the detection of small molecules, oligonucleotides, etc. The functionalized nanoparticles are selective and sensitive for the analytes, showing their great potential in biosensing. 


\section{Glossary}

Fluorescence (FL) is one of the most popular methods of detection in analytical chemistry because of its high sensitivity. There are still some well-known limitations. Most conventional fluorophores suffer from photobleaching, short lifetime, and limited brightness [45]. NPs of various materials have shown unique optical properties due to their large surface to volume ratio and unique electronic structure that has aided in the FL detection of various analytes. Fluorescent chemosensors are chemical systems that can detect and signal the presence of selected analytes through variations in their fluorescence emission. In its simplest design, a fluorescent chemosensor is composed of a fluorescent dye and a receptor, with a built-in transduction mechanism that converts recognition events into variations of the emission properties of the fluorescent dye. Nanoparticles have been used not only as betterperforming substitutes of traditional dyes but also as multivalent scaffolds for the realization of supramolecular assemblies, while their high surface to volume ratio allows for distinct spatial domains (bulk, external surface, pores and shells) to be functionalized to a comparable extent with different organic species.

Compared to organic dyes, fluorescent metal NPs are often stable and resistant to photobleaching and have higher luminescent intensity and longer lifetime. Most importantly, their emission is narrow and symmetrical and is tunable by size, shape, and composition [46]. Colloidal semiconductor quantum dots (QDs) have evolved beyond scientific novelties and are transitioning into bona fide analytical tools. It is well-known that metal nanoparticles (MNPs) can quench the fluorescence (FL) of fluorophores, but in recent years researchers have found that small metal NPs (or clusters) exhibit fluorescence. Accordingly, these NPs have been employed in sensing and imaging applications.

Lan et al. [47] used fluorescent water-soluble DNA-Ag nanoclusters (NCls) for $\mathrm{Cu}^{2+}$ ion detection [47]. A FL enhancement occurred for these probes in the presence of $\mathrm{Cu}^{2+}$, making them highly sensitive. In the presence of $\mathrm{Cu}^{2+}$ ions, the DNA becomes more rigid and as a result, the DNA protects the $\mathrm{Cu}^{2+}$-DNA-AgNCls more efficiently from environmental quenching. These are highly selective to $\mathrm{Cu}^{2+}$ ions compared to other metal ions ions by a factor of 350 -fold. The effect of $\mathrm{pH}$ on $\mathrm{Cu}^{2+}$ detection was relatively small but optimal at $\mathrm{pH}$ 6.0. $\mathrm{Cu}(\mathrm{OH})_{2}$ formed at higher $\mathrm{pH}$, which resulted in less DNA-Cu/AgNCls and lower FL enhancement. A decrease in the negative charge density on DNA at lower $\mathrm{pH}$ also hindered $\mathrm{Cu}^{2+}$ ion binding to the $\mathrm{NCl}$ surface, which resulted in lower FL. Reaction times were shorter at $60^{\circ}$ and $80^{\circ} \mathrm{C}$ but DNA-Cu/AgNCls were also unstable and a lower fluorescence signal appeared at these temperatures due to the uncontrolled reduction of $\mathrm{Cu}^{2+}$ ions and increased collisions with NCls. At temperatures higher than 80 ${ }^{\circ} \mathrm{C}$, the folded structure of DNA is less stable, which results in the decreased capping ability of DNA.

Fluorescent resonance energy transfer (or Förster resonance energy transfer, FRET) is a process involving the distance dependent energy transfer from a donor molecule to an acceptor molecule through dipole-dipole interactions [48]. The energy transfer rate is inversely proportional to $\mathrm{R}^{6}$, where $\mathrm{R}$ is the distance between the donor and acceptor. Usually fluorescent NPs serve as the FRET donor and a molecular dye serves as the acceptor. The close proximity of the acceptor to the NPs decreases the emission intensity of the NPs and increases the acceptor emission intensity [49].

Nanoparticle/nanowire assemblies with a degree of radial organization were prepared around luminescent semiconducting CdTe nanowires using bioconjugation with streptavidin and D-biotin linkers. Red-emitting nanowires $(\sim 6.62 \mathrm{~nm}$ diameter, $\sim 512 \mathrm{~nm}$ length) and green-emitting nanoparticles $(\sim 3.2 \mathrm{~nm}$ diameter $)$ were surface-modified with biotin, while orange-emitting nanoparticles ( $\sim 4.1 \mathrm{~nm}$ diameter) were decorated with streptavidin. CdTe nanocrystals produced two fuzzy layers around the nanowires in which the diameter of CdTe nanoparticles decreased with the distance from the nanowire axis. Förster resonance energy transfer (FRET) from the outside layer of nanoparticles to the central nanowire was observed for nanowires conjugated with $4.1 \mathrm{~nm}$ $\mathrm{CdTe}$. Addition of $3.2 \mathrm{~nm}$ CdTe resulted in a red-orange-green optical progression with band gaps of CdTe decreasing toward the axis of the superstructure. In this case, 4-fold luminescence enhancement of the nanowire luminescence was observed and was attributed to multistep FRET. This observation indicated the accumulation of photogenerated excitons in the cascade terminal.

In the FRET, the sensitivity is compromised by the background autofluorescence of the acceptor and other fluorophores within the sample. The nanosecond lifetimes of common fluorophores, including organic dyes and biocompounds, is small compared to the millisecond lifetimes of upconverting nanoparticles (UCNPs). Accordingly, the long emission lifetime of UCNPs should prolong the emission of the FRET signal from an acceptor. Time-resolved FRET (TR-FRET) can eliminate the background FL interference by setting an appropriate delay and gate time based on the different lifetimes [50]. The researchers [50] reported the first TR-FRET biosensing experiment based on aminefunctionalized lanthanidedoped NaYF4 NPs. A general and versatile biomimetic approach to synthesize water dispersible and functionalizable UCNPs for selective imaging of live cancer cells is reported.

The exceptional quenching ability of metallic nanoparticles makes them excellent materials for FRET-based biosensors, [51] for example, for the fabrication of molecular beacons for sensing DNA [52]. Here we describe a hybrid material composed of a single-stranded DNA (ssDNA) molecule, a $1.4 \mathrm{~nm}$ diameter gold nanoparticle, and a fluorophore that is highly quenched by the nanoparticle through a distance-dependent process. The fluorescence of this hybrid molecule increases by a factor of as much as several thousand as it binds to a complementary ssDNA. This composite molecule is a different type of molecular beacon with a sensitivity enhanced up to 100 -fold. 
Quantum Dots (QDs) are luminescent semiconductor nanocrystals with diameters of 2-10nm, composed of elements such as Cd, Zn, Se, Te, In, P, and/or As. It has been known for some time that QDs are fluorescent and that the emission wavelength is size tunable; it decreases with decreasing particle size due to an increase in the semiconductor band gap with decreasing size. QDs possess excellent photophysical properties and high quantum efficiency, inspiring researchers to broaden their application into many different areas, such as biological systems. The intrinsic FL of QDs made it possible in sensing applications by directly measuring the change in the FL intensity of the QDs due to their interaction with analytes [53,54]. Highly luminescent semiconductor quantum dots (zinc sulfide-capped cadmium selenide) have been covalently coupled to biomolecules for use in ultrasensitive biological detection. In comparison with organic dyes such as rhodamine, this class of luminescent labels is 20 times as bright, 100 times as stable against photobleaching, and one-third as wide in spectral linewidth. These nanometer-sized conjugates are water-soluble and biocompatible. Quantum dots that were labeled with the protein transferrin underwent receptor-mediated endocytosis in cultured HeLa cells, and those dots that were labeled with immunomolecules recognized specific antibodies or antigens [54].

QDs are also excellent substitutes over traditional FRET based dyes because of their excellent photophysical properties. The broad absorption spectrum means that different colors of QDs can be excited by a single excitation source as compared to traditional dye, which has to be excited within a narrow absorption band. This property together with the narrow symmetrical emission band makes QDs ideal for multiplexed optical sensing, especially for biological and medical applications. With multiplex sensing, several parameters can be determined simultaneously with a single measurement, significantly decreasing the time and cost associated with the measurement. The conjugation of QDs with highly ionspecific DNAzymes as a FRET sensor was recently demonstrated for multiplexed detection of $\mathrm{Cu}^{2+}$ and $\mathrm{Pb}^{2+}$ [55]. In this paper, the authors used silica-modified CdSe/ZnS core/shell QDs. The silica layer prevented nonspecific binding of heavy metal ions to the $\mathrm{QD}$, which can quench the FL. DNAzymes were attached to the QD through a zero length cross-linker, and both substrate and DNAzymes were labeled with a quencher. In the absence of the target metal ion, the quencher on the DNAzymes is in close proximity to the QD. Binding of the DNAzymes with the target metal ion causes the substrate to cleave off from the $\mathrm{QD}$, restoring the FL of the QD. The authors found that the FL intensity increased linearly for $\mathrm{Cu}^{2+}$ and $\mathrm{Pb}^{2+}$ in the concentration range of $1-50 \mathrm{nM}$, with a detection limit of 0.5 and $0.2 \mathrm{nM}$, respectively. They also demonstrated multiplexed sensing of $\mathrm{Cu}^{2+}$ and $\mathrm{Pb}^{2+}$, since they have different emission wavelengths of 625 and $530 \mathrm{~nm}$, respectively.

The authors developed highly sensitive and specific nanosensors based on quantum dots (QDs) and DNAzyme for multiplexed detection of heavy metal ions in liquid. The QDs were coated with a thin silica layer for increased stability and higher quantum yield while maintaining a relatively small size for highly efficient energy transfer. The QD-DNAzyme nanosensors were constructed by conjugating quencher-labeled DNAzymes onto the surface of carboxyl-silanized QDs. In the presence of metal ions, the emission is restored due to the cleavage of DNAzymes. The detection could be completed with a single laser excitation source. Multiplexed detection was also demonstrated using two different colors of QDs, showing negligible cross-talk between the $\mathrm{Pb}^{2+}$ detection and $\mathrm{Cu}^{2+}$ detection [55]. QDs not only have an intrinsic FL signal themselves but also the ability to enhance the FL signal of nearby fluorophores. There are some sensing and imaging applications based on QDamplified FL. The $\mathrm{Cd}^{2+}$ ions that are dissociated from a QD trigger the FL emission of nonfluorescent dye, such as Fluo-4 and Rhod-5N upon binding [56]. On the basis of this fact, Han et al. [57] designed a signal amplification of the FL of Rhod-5N for the in situ monitoring of carbohydrates in living cells.

\section{Surface-Enhanced Raman Spectroscopy (SERS)}

It was observed that certain molecules scatter a small fraction of light which is different from the energy of the incident beam [58]. The scattered photons either gain or lose energy due to the vibrational and rotational modes of the molecules, which is known as Raman scattering, and the resulting Raman spectrum (scattering intensity vs wavelength) is characteristic of the chemical structure of the molecule. The strength of the Raman signal is weak as only about 1 in $10^{6}-10^{10}$ photons are scattered inelastically [58].

Later, researchers discovered an enhancement in Raman scattering for molecules placed near a roughened surface, which is now known as surface-enhanced Raman spectroscopy (SERS). Jeanmaire and Van Duyne recognized SERS and reported the mechanism of enhancement based on the now well-known electromagnetic effect and chemical effect, respectively [59]. The fabrication of different SERS substrates which displayed enhancement factors ranging from $10^{6}-10^{8}$ have been reported $[59,60]$. In this work the authors have verified the remarkable sensitivity of Raman spectroscopy for the study of adsorbed pyridine on a silver surface, and extended its applicability to other nitrogen heterocycles and amines. New bands in the scattering spectrum of adsorbed pyridine have been characterized, as well as the Raman intensity response of all the surface pyridine bands as a function of electrode potential. As a result of these experiments, the authors have proposed a model of the adsorbed species for pyridine in which the adsorption is anion induced, leading to an axial end-on attachment to the electrode surface. The ability to obtain resonance Raman spectra with good signal-to-noise with laser powers less than $1.0 \mathrm{~mW}$, opens up possibilities of surface Raman studies with relatively inexpensive laser systems.

The electromagnetic field enhancement is due to the excitation of localized surface plasmons within the metal, which enhances the incident electric field intensity |E2| in the vicinity of the metals by $10^{2}$ to $10^{4}$ [61]. Molecules do not have to be in direct contact with the metal surface in order to experience the enhanced electric field intensity, but the molecules should be in close proximity of the metal surface (within $2-4 \mathrm{~nm}$ ) since the electromagnetic 
field decreases exponentially with an increase in the distance between the analyte and the metal surface [62]. In recent years, SERS has enjoyed a renaissance, and there is renewed interest in both the fundamentals and applications of SERS. New techniques for nanofabrication, the design of substrates that maximize the electromagnetic enhancement, and the discovery of single-molecule SERS are driving the resurgence of this field.

Metal nanoparticles with sharp features, that is, corners or edges, were found to act efficiently as SERS-active substrates, which is due to the local concentration of electromagnetic field. Odom and and coworkers reported a nanofabrication scheme for the synthesis of anisotropic and asymmetric gold nanoparticle pyramids [63]. This account explores nanofabricated pyramids, a new class of nanoparticles with tunable optical properties at visible and nearinfrared wavelengths. This system is ideally suited for designing multifunctional plasmonic materials for use in diagnostics, imaging, sensing, and therapeutics.

Surface Plasmon Resonance (SPR) is a versatile method for detecting changes in the refractive index occurring on thin metal films, usually gold, as a result of recognition events or chemical reactions [64]. Molecularly imprinted gold nanoparticles composites are generated on $\mathrm{Au}$-coated glass surfaces. The imprinting process involves the electropolymerization of thioaniline-functionalized AuNPs $(3.5 \mathrm{~nm})$ on a thioaniline monolayer-modified Au surface in the presence of a carboxylic acid, acting as a template analogue for the respective explosive. The binding of the analyte explosives to the AuNPs matrixes is probed by surface plasmon resonance spectroscopy, SPR, where the electronic coupling between the localized plasmon of the AuNPs and the surface plasmon wave leads to the amplification of the SPR responses originating from the dielectric changes of the matrixes upon binding of the different explosive materials. The resulting imprinted matrixes reveal high affinities and selectivity toward the imprinted explosives. Using citric acid as an imprinting template, AuNPs matrixes for the specific analysis of pentaerythritol tetranitrate (PETN) or of nitroglycerin (NG) were prepared, leading to detection limits of $200 \mathrm{fM}$ and $20 \mathrm{pM}$, respectively. Similarly, using maleic acid or fumaric acid as imprinting templates, high-affinity sensing composites for ethylene glycol dinitrate (EGDN) were synthesized, leading to a detection limit of 400fM for both matrixes [64].

By detecting a small refractive index change at the metal-analyte interface, the information of the molecular interactions can be obtained by measuring the optical characteristics (intensity, phase, and polarization) of light reflected from the optical setup, usually using a Kretschmann configuration [65]. SPR was first proposed for the direct monitoring of specific antibody-antigen interactions in the 1980s [66]. Since then, the technology has grown rapidly and resulted in a vast array of platforms available commercially or in researchlaboratory settings [67]. SPR sensing is a label-free and sensitive technique for the detection of molecular interactions. The advantages of this technique are well stated in a paper published by Gao et al. [68].
Localized Surface Plasmon Resonance (LSPR) refers to the collective oscillation of the conducting electrons of metal NPs when their frequency matches that of the incident electromagnetic radiation. It is one of the characteristic optical properties of metal NPs (MNPs) [69], and serves as the basis for SPR (although not localized) and surface-enhanced Raman spectroscopy (SERS). A strong absorption band(s) or increased scattering intensity of the radiation occurs at certain wavelengths for the MNPs as a result of this phenomenon [70]. Acording to Mie theory, LSPR of the NP is mainly related to the NP size, shape, composition, interparticle distance, and dielectric constant (refractive index) of the surrounding medium [62,71]. Recent developments have greatly improved the sensitivity of optical sensors based on metal nanoparticle arrays and single nanoparticles.

Noble metal NPs are one of the promising tools for optical bioimaging due to the strong light scattering at the LSPR frequency, typically in the visible range $[72,73]$. The scattering light intensity is sensitive to the size, shape, and interparticle distance of the NPs. Typically, NPs are labeled with specific receptors that interact with molecules in biological samples. Recent developments in photonic technology provide the ability to noninvasively image cells in vivo; these new cellular imaging technologies have the potential to dramatically improve the prevention, detection, and therapy of epithelial cancers. Endoscope-compatible microscopies, such as optical coherence tomography and reflectance confocal microscopy, image reflected light, providing a three-dimensional picture of tissue microanatomy with excellent spatial resolution (110 micro $\mathrm{m})$.

Gold nanoparticles with unique optical properties may be useful as biosensors in living whole cells. Using a simple and inexpensive technique, wthe authors recorded SPR scattering images and SPR absorption spectra from both colloidal gold nanoparticles and from gold nanoparticles conjugated to monoclonal anti-epidermal growth factor receptor (anti-EGFR) antibodies after incubation in cell cultures with a nonmalignant epithelial cell line (HaCaT) and two malignant oral epithelial cell lines (HOC 313 clone 8 and HSC 3). Colloidal gold nanoparticles are found in dispersed and aggregated forms within the cell cytoplasm and provide anatomic labeling information, but their uptake is nonspecific for malignant cells. The anti-EGFR antibody conjugated nanoparticles specifically and homogeneously bind to the surface of the cancer type cells with $600 \%$ greater affinity than to the noncancerous cells. This specific and homogeneous binding is found to give a relatively sharper SPR absorption band with a red shifted maximum compared to that observed when added to the noncancerous cells. These results suggest that SPR scattering imaging or SPR absorption spectroscopy generated from antibody conjugated gold nanoparticles can be useful in molecular biosensor techniques for the diagnosis and investigation of oral epithelial living cancer cells in vivo and in vitro [73].

The w/o microemulsion method offers a lot of advantages with respect to others, namely, the use of a simple equipment, 
homogeneous mixing, high degree of particles size and composition control, formation of metal NPs with high surface area and the used of soft condition of synthesis near ambient temperature and pressure [74] (Figure 3). The main strategy for the MNP synthesis in w/o microemulsion consists in mixing two microemulsions, one containing the metal precursor solution and the other one containing the reduction agend [75]. Upon mixing procedure, both reactants (metal precursor and reduction agent solutions) will contact each other due to the coalescence and droplet collisions and will react to form MNP which remaine confined to the interior of microemulsion droplets. Even through MNP obtained by w/o microemulsion shows superior properties and performance, at the industrial level this method has no good acceptance, mainly due to the large amount of solvents (oils) which represent the main component of these systems being the continuous phase [76,77].

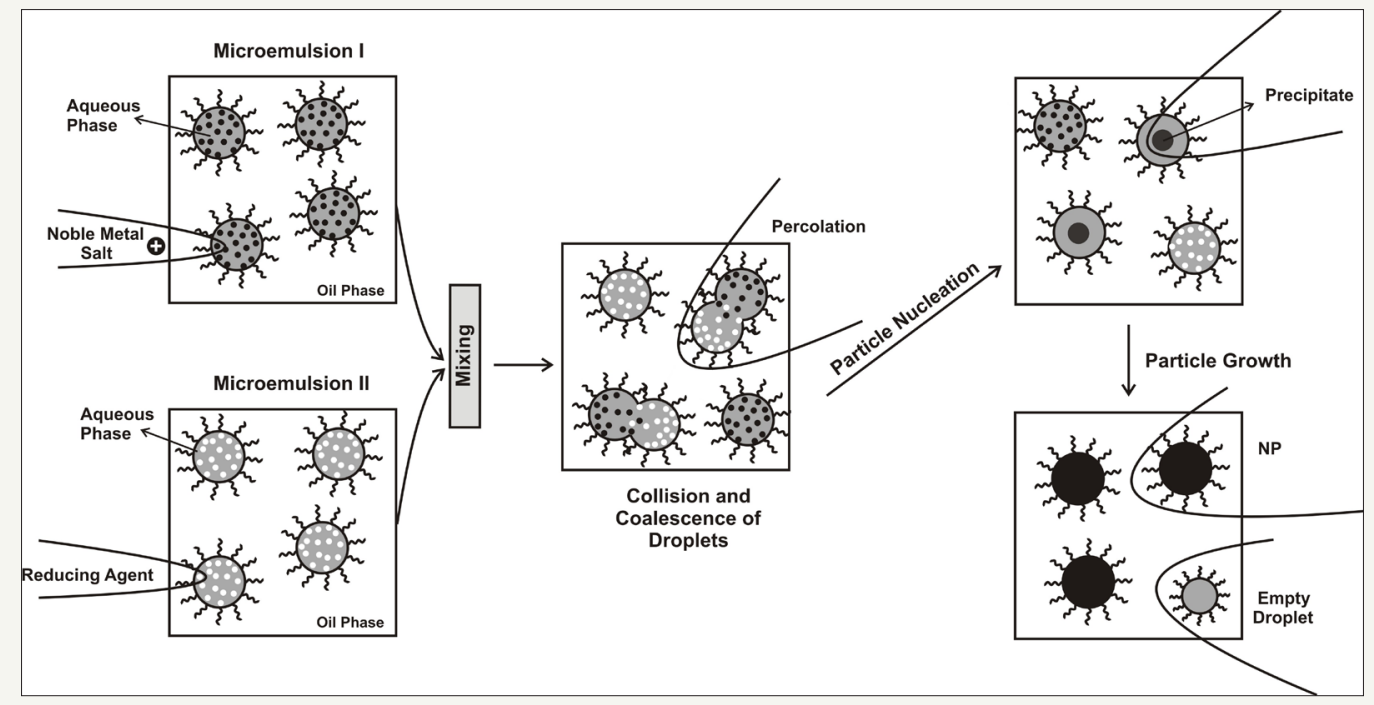

Figure 3: The schematic view of the synthesis of (noble) metal nanoparticles by reactions proceeding via microdroplets collisions [77].

Photothermal therapy is a minimally invasive, local treatment modality with minimal toxicity. It mainly depends on triggering a photosensitizer by an electromagnetic radiation, such as radio frequency, microwaves, NIR, or visible light in order to convert this energy into heat. In clinical settings, this process is called hyperthermia. Hyperthermia may trigger several hazardous phenomena, such as denaturation of proteins, lysis of cell membrane, evaporation of cytosol, thereby leading to cell death. Photothermal therapy, unlike photodynamic therapy which has an antitumor activity relying on the generation of radical oxygen species, hence, presence of oxygen is essential, mainly exerts its effect by increasing the temperature of milieu; therefore, it does not require oxygen in order to generate its cytotoxic effect on cancerous cells.

Photodynamic Therapy (PDT), is a form of phototherapy involving light and a photosensitizing chemical substance, used in conjunction with molecular oxygen to elicit cell death (phototoxicity). PDT has proven ability to kill microbial cells, including bacteria, fungi and viruses. It is used clinically to treat a wide range of medical conditions, including wet age-related macular degeneration, psoriasis, atherosclerosis and has shown some efficacy in anti-viral treatments, including herpes. It also treats malignant cancers including head and neck, lung, bladder and particular skin. The technology has also been tested for treatment of prostate cancer, both in a dog model and in human prostate cancer patients.

\section{Acknowledgement}

This research is supported by the VEGA project No. 2/0164/17.

\section{References}

1. Nguyen DT, Kim DJ, Kim KS (2011) Controlled synthesis and biomolecular probe application of gold nanoparticles. Micron 42: 207-227.

2. Parab H, Jung C, Woo MA, Park HG (2011) An anisotropic snowflake-like structural assemblyof polymer-capped gold nanoparticles. J Nanopart Res 13: 2173-2180.

3. Stober W, Fink A, Bohn E (1968) Controlled growth of monodisperse silica spheres in the micron size range. J Colloid Interface Sci 26: 62-69.

4. Wang XD, Shen ZX, Sang T, Cheng XB, Li MF, et al. (2010) Preparation of spherical silica particles by Stöber process with high concentration of tetra-ethyl-orthosilicate. J Colloid Interface Sci 341: 23-29.

5. Rieter WJ, Kim JS, Taylor KML, An H, Tarrant T, et al. (2007) Hybrid silica nanoparticles for multimodal imaging. Angew Chem Int Ed 46: 36803684.

6. Taylor-Pashow KML, Della RJ, Huxforda RC, Lin W (2010) Hybrid nanomaterials for biomedical applications. Chem Commun 46: 58325849.

7. Enrichi F, Riccò R, Meneghello A, Pierobon R, Cretaio E, et al. (2010) A Opt Mater 32: 1652-1658.

8. Bonacchi S, Genovese D, Juris R, Montalti M, Prodi L, et al. (2011) Angew Chem, Int Ed 250: 4056-4066.

9. Verhaegh NAM, Van BA (1994) Dispersions of rhodamine-labeled silica spheres: synthesis, characterization, and fluorescence confocal scanning laser microscopy. Langmuir 10: 1427-1438. 
10. Xue L, Li B, Fei Q, Feng G, Huan Y, et al. (2010) Carboxylate-modified squaraine dye doped silica fluorescent $\mathrm{pH}$ nanosensors. Nanotechnology 21: 215502.

11. Wang X, Ramstrom O, Yan M (2011) Dye-doped silica nanoparticles as efficient labels for glycans. Chem Commun 47: 4261-4263.

12. Lei J, Wang L, Zhang J (2010) Ratiometric pH sensor based on mesoporous silica nanoparticles and Förster resonance energy transfer. Chem Commun 46(44): 8445-8447.

13. Zong C, Ai K, Zhang G, Li H, Lu L (2011) Fluorescent graphene-like carbon nitrides. Anal Chem 83: 3126-3132.

14. Kim S, Ohulchanskyy TY, Pudavar HE (2007) Organically modified silica nanoparticles co-encapsulating photosensitizing drug and aggregationenhanced two-photon absorbing fluorescent dye aggregates for twophoton photodynamic therapy. J Am Chem Soc 129(9): 2669-2675.

15. He XX, Wu X, Wang KM (2009) Methylene blue-encapsulated phosphonate-terminated silica nanoparticles for simultaneous in vivo imaging and photodynamic therapy. Biomaterials 30(29): 5601-5609.

16. Lin PY, Le GY, Chiu WI, Jian RS, Lu CJ (2019) A single light spot GC detector employing localized surface plasmon resonance of porous Au@ $\mathrm{SiO}_{2}$ nanoparticle multilayer. Analyst 144(2): 698-706.

17. Roy I, Ohulchanskyy TY, Bharali DJ (2005) Optical tracking of organically modified silica nanoparticles as DNA carriers: a nonviral, nanomedicine approach for gene delivery. Proc Natl Acad Sci USA 102(2): 279-284.

18. Bharali DJ, Klejbor I, Stachowiak EK (2005) Organically modified silica nanoparticles - a novel non-viral vector for in vivo gene delivery and expression in the brain. Proc Natl Acad Sci USA 102(32): 11539-11544.

19. Yan E, Ding Y, Chen C (2009) Polymer/silica hybrid hollow nanospheres with $\mathrm{pH}$-sensitive drug release in physiological and intracellular environments. Chem Commun 19: 2718-2720.

20. Corma A, Diaz U, Arrica M (2009) Organic-inorganic nanospheres with responsive molecular gates for drug storage and release. Angew Chem Int Ed 48(34): 6247-6250.

21. Bagwe RP, Yang C, Hilliard LR (2004) Optimization of dye-doped silica nanoparticles prepared using a reverse microemulsion method. Langmuir 20(19): 8336-8342.

22. Slowing I, Trewyn BG, Lin VSY (2007) Mesoporous silica nanoparticles for intracellular delivery of membrane-impermeable proteins. J Am Chem Soc 129: 8845-8849.

23. Mortera R, Vivero EJL, Slowing I, Garrone E, Onida B, et al. (2009) Cellinduced intracellular controlled release of membrane impermeable cysteine from a mesoporous silica nanoparticles-based drug delivery system. Chem Commun19: 3219-3221.

24. Lai CY, Trewyn BG, Jeftinija DM, Jeftinija K , Xu S, et al. (2003) A mesoporous silica nanosphere-based carrier system with chemically removable CdS nanoparticle caps for stimuli-responsive controlled release of neurotransmitters and drug molecules. J Am Chem Soc 125(15): 4451-4459.

25. Kim J, Kim HS, Lee N, Kim T, Kim H, et al. (2008) Multifunctional uniform nanoparticles composed of a magnetite nanocrystal core and a mesoporous silica shell for magnetic resonance and fluorescence imaging and for drug delivery. Angew Chem Int Ed 47(44): 8438-8441.

26. Liong M, Lu J, Kovochich M, Xia T, Ruehm SG, et al. (2008) Multifunctiona inorganic nanoparticles for imaging, targeting, and drug delivery. ACS Nano 2(5): 889-896.

27. Lai CW, Wang YH, Lai CH, Yang MJ, Chen CY, et al. (2008) Iridiumcomplex-functionalized $\mathrm{Fe}_{3} \mathrm{O}_{4} / \mathrm{SiO}_{2}$ core/shell nanoparticles: a facile three-in-one system in magnetic resonance imaging, luminescence imaging, and photodynamic therapy. Small 4(2): 218-224.
28. Ye C, Li M, Xue M, Shen W, Cao T, et al. (2011) Rhodamine B coated AuNP arrays. Mater Chem 21: 5234-2539.

29. Kelly KL, Coronado E, Zhao LL, Schatz GC (2003) The optical properties of metal nanoparticles: The influence of size, shape, and dielectric environment. J Phys Chem B 107: 668-677.

30. Nuzzo RG, Allara DL (1983) Adsorption of bifunctional organic disulfides on gold surfaces. J Am Chem Soc 105: 4481-4483.

31. Ulman A (1996) Formation and structure of self-assembled monolayers. Chem Rev 96: 1533-1554.

32. Xia Y, Rogers JA, Paul KE, Whitesides GM (1999) Unconventional methods for fabricating and patterning nanostructures. Chem Rev 99: 1823-1848.

33. Ferrari M (2005) Cancer nanotechnology: opportunities and challenges. Nat Rev Cancer 5(3): 161-171.

34. Brown RJC, Milton MJT (2008) Surface enhanced Raman spectroscopy for chemical analysis. J Raman Spectrosc 39: 1313-1326.

35. Lochner N, Lobmaier C, Wirth M, Leitner A, Pittner F, (2003) Enhancing the analytical performance of immunoassays that employ metalenhanced fluorescence. Eur J Pharm Biopharm 56: 469-477.

36. Datar RH, Richard JC (2010) Nanomedicine: concepts, status and the future. Medical Innovation \& Business 2: 6-17.

37. Kim C, Ghosh P, Rotello VM (2009) Multimodal drug delivery using gold nanoparticles. Nanoscale 1: 61-7.

38. Kawasaki ES, Player A (2005) Nanotechnology, nanomedicine, and the development of new, effective therapies for cancer. Nanomedicine: Nanotechnology, Biology and Medicine 1: 101-109.

39. Saini R, Lee N, Liu K, Poh C (2016) Prospects in the application of photodynamic therapy in oral cancer and premalignant lesions. Cancers 8(9): E83.

40. Huang X, El-Sayed I, Qian W, El-Sayed M (2006) Cancer cell imaging and photothermal therapy in the near-infrared region by using gold nanorods. J Am Chem Soc 128(6): 2115-2120.

41. PK Jain, X Huang, Ivan H, El-Sayed, Mostafa A, et al. (2007) Review of some interesting surface plasmon resonance-enhanced properties of noble metal nanoparticles and their applications to biosystems. Plasmonics 2(3): 107-118.

42. Jain PK, Huang X, El-Sayed IH, El-Sayed MA (2008) Noble metals on the nanoscale: optical and photothermal properties and some applications in imaging, sensing, biology and medicine. Acc Chem Res 41 (12): 15781586

43. Huang X, El-Sayed IH, Qian W, El-Sayed MA (2006) Cancer cell imaging and photothermal therapy in the near-infrared region by using gold nanorods. J Am Chem Soc 128 (6): 2115-2120.

44. Qiang W, Hui Li, D Xu (2013) Metal enhanced fluorescent biosensing assays for DNA through the coupling of silver nanoparticles. Anal Methods 3: 569-812.

45. Bau L, Tecilla P, Mancin F (2011) Sensing with fluorescent nanoparticles. Nanoscale 3(1): 121-133

46. Algar WR, Susumu K, Delehanty JB, Medintz IL (2011) Semiconductor quantum dots in bioanalysis: crossing the valley of death. Anal Chem 83(23): 8826-8837.

47. Lan GY, Huang CC, Chang HT (2010) Silver nanoclusters as fluorescent probes for selective and sensitive detection of copper ions.Chem Commun 46(8): 1257-1259.

48. Lee J, Govorov AO, Kotov NA (2005) Bioconjugated superstructures of CdTe nanowires and nanoparticles: Multistep cascade Förster resonance energy transfer and energy channeling. Nano Lett 5: 2063-2069. 
49. Jares EEA, Jovin TM (2003) FRET imaging. Nat Biotechnol 21(11): 1387 95.

50. Tu D, Liu L, Ju Q Liu Y, Zhu H, et al. (2011) A illustration of functionalized upconversion nanoparticle (f-UCNP). Angew Chem Int Ed 50: 63066310 .

51. Sapsford KE, Berti L, Medintz IL (2006) Materials for fluorescence resonance energy transfer analysis: beyond traditional donor-acceptor combinations. Angew Chem Int Ed 45(28): 4562-4589.

52. Dubertret B, Calame M, Libchaber AJ (2001) Single-mismatch detection using gold-quenched fluorescent oligonucleotides. Nat Biotechnol 19(4): 365-370.

53. Bruchez M, Jr Moronne M, Gin P, Weiss S, Alivisatos AP (1998) Semiconductor nanocrystals as fluorescent biological labels. Science 281(5385): 2013-2016.

54. Chan WCW, Nie S (1998) Quantum dot bioconjugates for ultrasensitive nonisotopic detection. Science 281(5385): 2016-2018.

55. Wu CS, Oo MKK, Fan X (2010) Highly sensitive multiplexed heavy metal detection using quantum-dot-labeled DNAzymes. ACS Nano 4(10): 5897-5904.

56. Li J, Zhang T, Ge J, Yin Y, Zhong W (2009) Fluorescence signal amplification by cation exchange in ionic nanocrystals. Angew Chem Int Ed 48(9): 1588-1591.

57. Han E, Ding L, Ju H (2011) Highly sensitive fluorescent fnalysis of dynamic glycan expression. Anal Chem 83(18): 7006-7012.

58. Raman CV, Krishnan KS (192) A new type of secondary radiation. Nature 121: 501-502.

59. Jeanmaire DL, Van Duyne RPJ (1977) Surface raman spectroelectrochemistry. Part I. Heterocyclic, aromatic, and aliphatic amines adsorbed on the anodized silver electrode. Electroanal Chem 84(1): 1-20.

60. Kneipp J, Kneipp H, Rajadurai A, Redmond RW, Kneipp K (2009) Optical probing and imaging of live cells using SERS labels. J Raman Spectrosc 40(1): 1-5.

61. Van Duyne RP, Zou SL (2005) Plasmonic materials for surface-enhanced sensing and spectroscopy. MRS Bull 30: 368-375.

62. Camden JP, Dieringer JA, Zhao J, Van Duyne RP (2008) Controlled plasmonic nanostructures for surface-enhanced spectroscopy and sensing. Acc Chem Res 41(12): 1653-1661.

63. Lee J, Hasan W, Stender CL, Odom TW (2008) Pyramids: a platform for designing multifunctional plasmonic particles. Acc Chem Res 41(12): 1762-1771.

64. Riskin M, Tel-Vered R, Willner I (2010) Molecularly imprinted Au nanoparticles composites on $\mathrm{Au}$ surfaces for the surface plasmon resonance detection of pentaerythritol tetranitrate, nitroglycerin, and ethylene glycol dinitrate. Adv Mater 22: 1387-1391.
65. Homola J (2008) Surface plasmon resonance sensors for detection of chemical and biological species. Chem Rev108(2): 462-493.

66. Flanagan MT, Pantell RH (1984) Surface plasmon resonance and immunosensors. Electron Lett 20: 968-970.

67. Situ C, Mooney MH, Elliott CT, Buijs J (2010) TrAC. Advances in surface plasmon resonance biosensor technology towards high-throughput food-safety. Trends Anal Chem 29(1): 1305-1315.

68. Gao S, Koshizaki N, Tokuhisa H, Koyama E, Sasaki T, et al. (2010) The surface plasmon resonance (SPR) of the metal nanoparticles. Adv Funct Mater 20: 78-86.

69. Lopatynskyi AM, Lopatynska OG, Guo LJ, Chegel VI (2011) Localized surface plasmon resonance in Au nanoprisms on glass substrates. IEEE Sens J 11: 361-369

70. Beeram SR, Zamborini FP (2011) Effect of protein binding coverage, location, and distance on the localized surface plasmon resonance response of purified au nanoplates grown directly on surfaces. J Phys Chem C 115(15): 7364-7371.

71. Anker JN, Hall WP, Lyandres O, Shah NC, Zhao J, et al. (2008) Biosensing with plasmonic nanosensors. Nat Mater 7(6): 442-453

72. Sokolov K, Follen M, Aaron J, Pavlova I, Malpica A, et al. (2003) Real-time vital optical imaging of precancer using anti-epidermal growth factor receptor antibodies conjugated to gold nanoparticles. Cancer Res 63(9): 1999-2004.

73. El-Sayed IH, Huang X, El-Sayed MA (2005) Surface plasmon resonance scattering and absorption of anti-EGFR antibody conjugated gold nanoparticles in cancer diagnostics: applications in oral cancer. Nano Lett 5(5): 829-834

74. Sanchez DM, Pemartin K, Boutonnet M (2012) Preparation of inorganic nanoparticles in oil-in-water microemulsions: A soft and versatile approach. Curr Opin Colloid Interface Sci 17(5): 297-305.

75. Thikett K, Brice H, Myakonkaya O, Eastoe J, Rogers SE, (2010) Microemulsion-based organogels containing inorganic nanoparticles. Soft Matter 6: 1291-1296

76. Boutonnet M, Lögdberg S, Svensson E (2008) Recent developments in the application of nanoparticles prepared from w/o microemulsions in heterogeneous catalysis. Curr Opin Colloid Interface Sci 13(4): 270-286.

77. Capek I (2017) Noble metal nanoparticles, Preparation, Composite nanostructures, Biodecoration and Collective properties, Series Edn. In: DJ Lockwood, Capek I (Eds.), Nanostructure science and technology, Springer, Tokyo, Japan, pp. 1-554.
Creative Commons Attribution 4.0 International License

For possible submissions Click Here

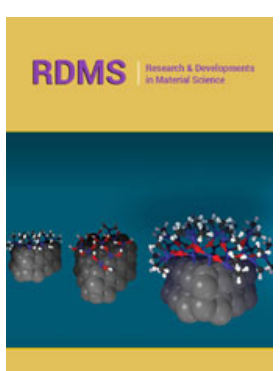

Research \& Development in Material Science

\section{Benefits of Publishing with us}

- High-level peer review and editorial services

- Freely accessible online immediately upon publication

- Authors retain the copyright to their work

- Licensing it under a Creative Commons license

- Visibility through different online platforms 